\title{
Chronic Thromboembolic Pulmonary Hypertension Associated with Chronic Inflammation
}

\author{
Naoyuki Kuse ${ }^{1,2}$, Shinji Abe ${ }^{1}$, Hidehiko Kuribayashi ${ }^{1}$, Asami Fukuda ${ }^{1}$, Yuji Kusunoki ${ }^{1}$, \\ Ritsuko Narato ${ }^{1}$, Hitoshi Saito ${ }^{1}$ and Akihiko Gemma ${ }^{2}$
}

\begin{abstract}
Chronic thromboembolic pulmonary hypertension (CTEPH) is one of the leading causes of severe pulmonary hypertension. According to previously reported studies in the pertinent literature, chronic inflammatory conditions may be implicated in the development of CTEPH. We herein describe the case of a 56-year-old woman who was diagnosed with CTEPH in association with chronic infection. The patient had experienced five episodes of pneumonia in the five years prior to the diagnosis of CTEPH. Blood tests from the previous five years of outpatient follow-up demonstrated that the C-reactive protein level was slightly elevated. This case suggests that a relationship exists between chronic inflammation and CTEPH, and furthermore, may contribute towards elucidating the pathophysiology of CTEPH.
\end{abstract}

Key words: chronic thromboembolic pulmonary hypertension, chronic inflammation, Staphylococcal species infection, C-reactive protein

(Intern Med 55: 1471-1476, 2016)

(DOI: 10.2169/internalmedicine.55.5146)

\section{Introduction}

Chronic thromboembolic pulmonary hypertension (CTEPH) is a common form of pulmonary hypertension $(\mathrm{PH})$, which was previously thought to be caused by insoluble blood clots that obstruct the pulmonary vessel $(1,2)$. CTEPH was formerly attributed to the formation of single or recurrent pulmonary emboli that potentially arise from deep vein thrombosis. However, more recently, it has been found that both the symptoms of pulmonary embolism and classic thromboembolic risk factors are lacking in patients with CTEPH (3). Although the etiology remains unclear, factors such as immune phenomena and inflammation have been linked to CTEPH (2). In this case report, we describe a woman with CTEPH, which was presumably associated with chronic inflammation, including active infectious episodes.

\section{Case Report}

A 56-year-old Pakistani woman was admitted to our hos- pital with a primary complaint of worsening dyspnea. An examination of her medical history revealed a lung disease of unknown cause during childhood. The patient was diagnosed with bronchial asthma and inhalation of steroids with a long-acting bronchodilator was initiated. Over the last five years, five episodes of pneumonia that required hospitalization had been documented. At the first hospitalization, chest radiographs and computed tomography (CT) scans demonstrated consolidation in the right lower lung field without cardiomegaly or dilation of the pulmonary artery (Fig. 1a, b). Electrocardiography (ECG) showed the presence of isolated sinus tachycardia. The ensuing pneumonia episodes were treated with antibiotics, which improved the patient's clinical condition, including her laboratory data and radiological findings. The effectiveness of antibiotic therapy was in accordance with the detection of Staphylococcus aureus in sputum cultures from three of the five episodes of pneumonia. Subsequently, long-term oxygen therapy was administered because of chronic respiratory failure. The patient had no history of abortion, smoking, drug abuse, or blood transfusion. On this hospital admission, her vital signs were

\footnotetext{
${ }^{1}$ Department of Pulmonary Medicine, Tokyo Metropolitan Hiroo General Hospital, Japan and ${ }^{2}$ Department of Pulmonary Medicine and Oncology, Graduate School of Medicine, Nippon Medical School, Japan

Received for publication February 12, 2015; Accepted for publication July 28, 2015

Correspondence to Dr. Shinji Abe, sabe@nms.ac.jp
} 


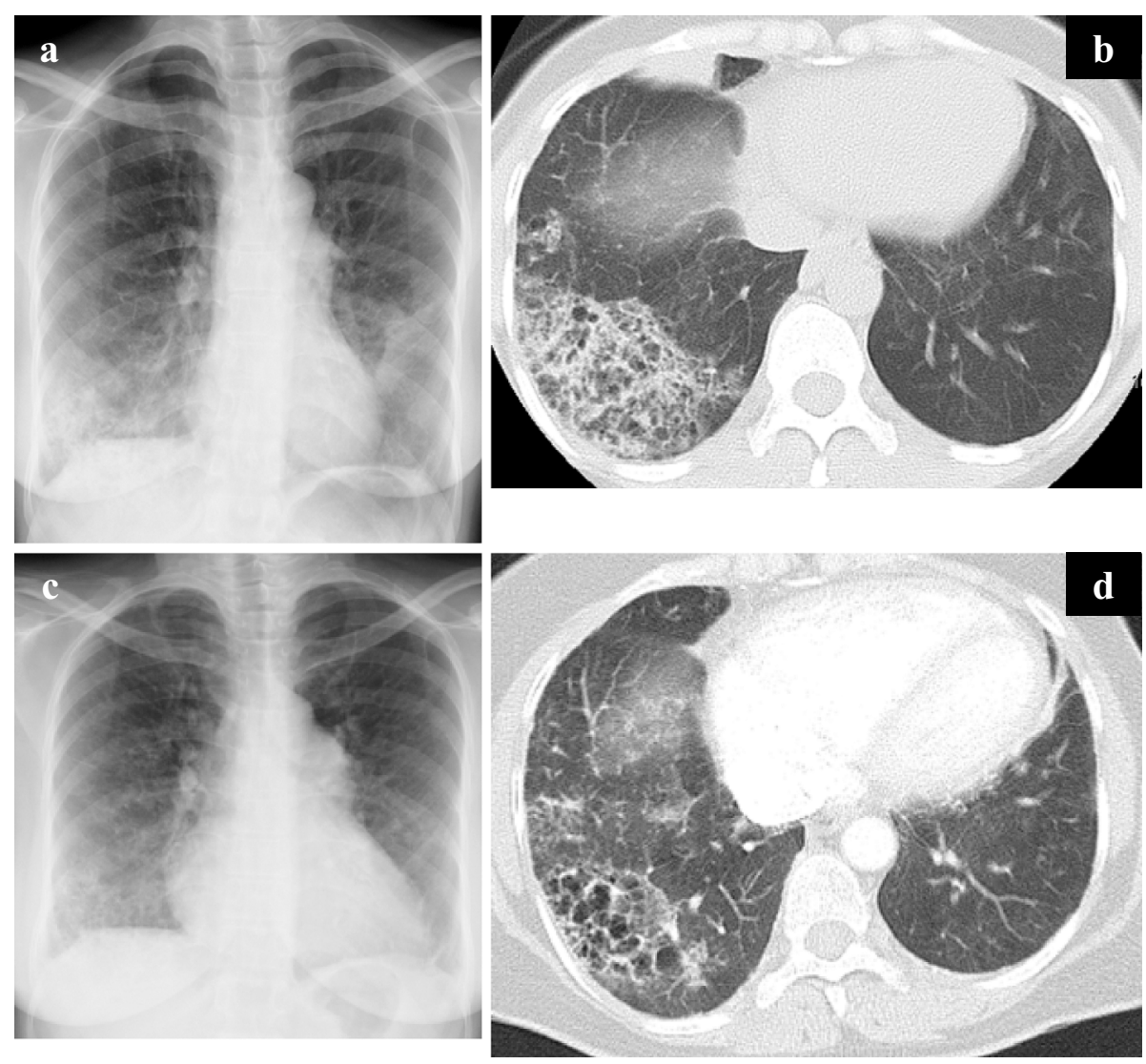

Figure 1. (a) Chest radiograph from five years previously, at the time of the patient's first hospitalization for pneumonia. In the right lower lung field, consolidation was observed. (b) Contrastenhanced CT at the first hospitalization for pneumonia. The architecture of the right lung lobe was disrupted and consolidation was present. (c) A chest radiograph at the most recent hospital admission. Cardiomegaly and moderate enlargement of the right atrium were revealed compared with the previous results. In the right lower lung field, a reticular shadow was shown. (d) Chest CT at the most recent admission. No consolidation was seen in the right lung lobe.

as follows: temperature, $36.2^{\circ} \mathrm{C}$; blood pressure, $118 / 58$ $\mathrm{mmHg}$; heart rate of $96 \mathrm{bpm}$; and oxygen saturation, $83 \%$ under $3 \mathrm{~L} / \mathrm{min}$ of oxygen. On physical examination, orthopnea was detected, normal S1 and loud P2, and wheezing in both lung fields on chest auscultation. The chest radiographs and CT scans showed cardiomegaly and moderate enlargement of the right atrium compared with five years previously (Fig. 1c, d). An ECG indicated right axis deviation, Rwave progression in V1 and V2, inverted T-waves in V1 to V4, and S-wave increases in V5 and V6 compared with the previous results. Transthoracic echocardiography confirmed severe $\mathrm{PH}$ with a predicted right ventricular systolic pressure (RVSP) of $111.7 \mathrm{mmHg}$.

The laboratory tests showed a slightly elevated white blood cell count $(9,800$ cells $/ \mu \mathrm{L})$ without eosinophilia, and high levels of C-reactive protein (CRP: $1.9 \mathrm{mg} / \mathrm{dL}$ ) and brain natriuretic peptide (BNP: $545.6 \mathrm{pg} / \mathrm{mL}$ ).The D-dimer levels were also moderately elevated $(2.3 \mu \mathrm{g} / \mathrm{mL})$. Thrombophilia tests, including the activated protein $\mathrm{C}$ ratio, activated protein $\mathrm{S}$ ratio, factor $\mathrm{V}$ Leiden, and antiphospholipid antibodies were normal. An examination of autoantibody titers, including anti-ribonucleoprotein antibody, anti-Smith anti- body, anti-Ro/SSA antibodies and anti-La/SSB antibodies, as well as anti-centromere antibody, anti-topoisomerase I antibody, and serum complement, demonstrated that all were within the normal ranges, except for a low titer of antinuclear antibody (ANA; 1:40). The positive ANA result was deemed to be nonspecific because none of the other clinical or laboratorial findings were indicative of collagen vascular disease. An arterial blood gas analysis under $3 \mathrm{~L} / \mathrm{min}$ of oxygen suggested respiratory acidosis $\left(\mathrm{pH}:\right.$ 7.346, $\mathrm{PaO}_{2}$ : 74.5 Torr, $\mathrm{PaCO}_{2}$ : 77.6 Torr, $\left.\mathrm{HCO}_{3}: 41.5 \mathrm{mmol} / \mathrm{L}\right)$. Contrastenhanced CT scans from the chest to the inferior limb were performed. Mosaic perfusion was observed in both lung fields (Fig. 2a) and the lung parenchyma of the right lower lobe was disrupted (Fig. 2b). Intraluminal filling defects were found at the segment branch of the right lower lobe pulmonary artery, however, no other clots were detected in either lung field (Fig. 2c). In addition, deep vein clots and other abnormalities were not present in the vessels and pelvis. Ventilation-perfusion lung scintigraphy revealed normal ventilation scans, but several subpleural wedge-shaped perfusion defects (Fig. 3). Pulmonary angiography showed abrupt narrowing in the right middle and lower pulmonary 

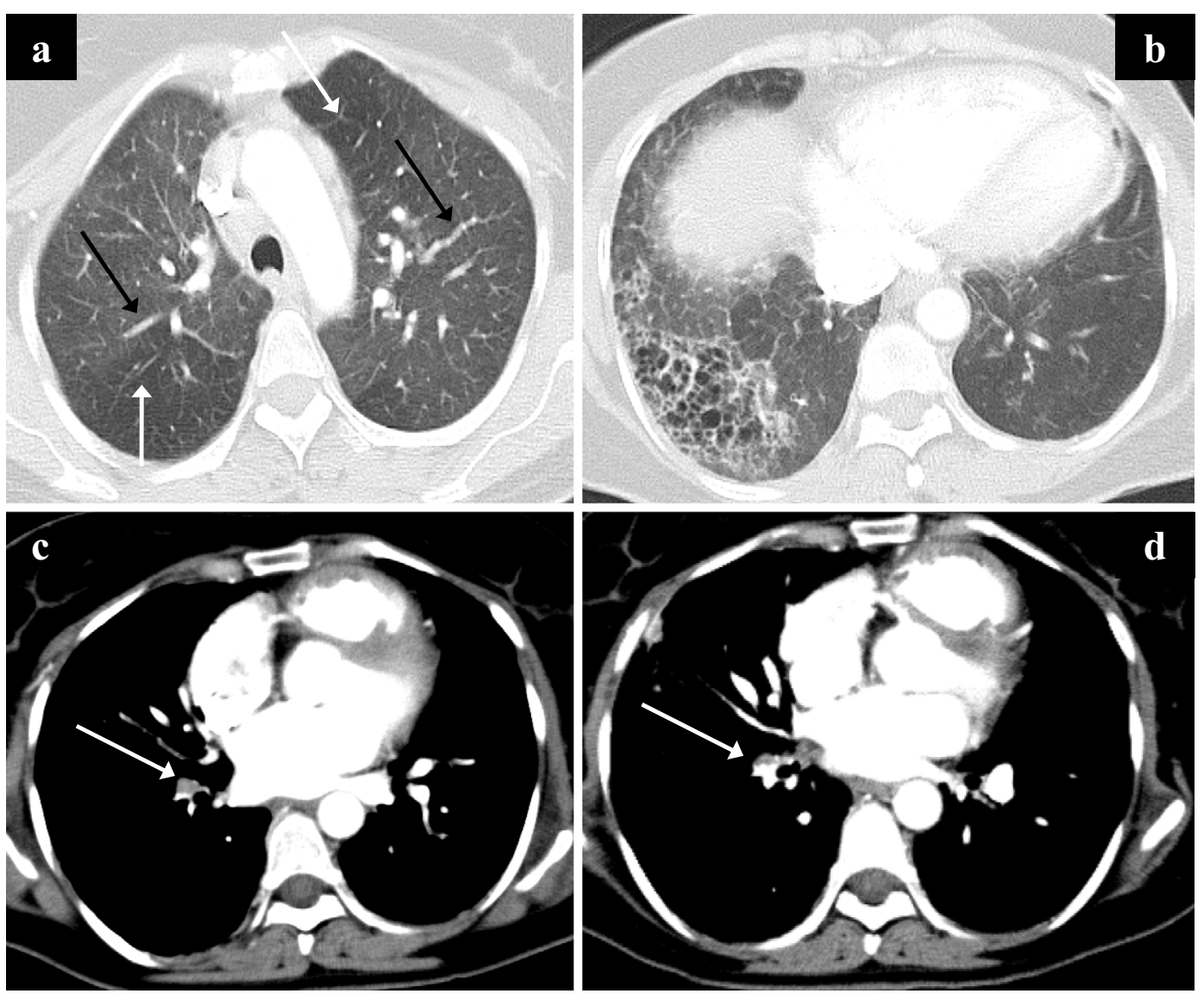

Figure 2. Contrast-enhanced CT scans at the most recent admission (a-c) and after approximately six months of anticoagulation therapy (d). (a) Mosaic perfusion was observed in both lung lobes. Vessels in the high attenuation areas are prominent (black arrows), whereas vessels in the low attenuation areas (white arrows) are smaller. (b) The architecture of the right lung lobe was disrupted. (c, d) The obstruction caused by the blood clot was visualized in the pulmonary artery vessels. The obstruction remained over the course of six-month anticoagulation treatment (white arrow).

vessels as well as obstruction of the left lower vessels (Fig. 4). Right heart catheterization demonstrated a mean right arterial pressure of $5 \mathrm{mmHg}$, mean pulmonary arterial pressure of $62 \mathrm{mmHg}$, cardiac output of $2.53 \mathrm{~L} / \mathrm{min} / \mathrm{m}^{2}$, pulmonary wedge pressure of $14 \mathrm{mmHg}$ and pulmonary vascular resistance of 1,211 dynes.s. $\mathrm{cm}^{-5}$. Subsequent coronary angiography did not reveal any abnormalities. These clinical data were indicative of worsening PH associated with an established chronic pulmonary embolism. Treatment with the anticoagulant warfarin (2 $\mathrm{mg} /$ day) was initiated and lowdose furosemide was administered to control the symptoms of heart failure. Following the approval of subsidies by the Japanese-specified disease treatment research program, beraprost $(60 \mu \mathrm{g} /$ day $)$ and sildenafil $(60 \mathrm{mg} /$ day $)$ were added. After six months of anticoagulant therapy, transthoracic echocardiography showed that the predicted RVSP was $102.0 \mathrm{mmHg}$, while the contrast-enhanced CT scans showed mural defects and abrupt narrowing (Fig. 2d). Therefore, the diagnosis of CTEPH was confirmed. However, even at one year prior to hospitalization for worsening dyspnea, the patient's lung function was remarkably poor [vital capacity (VC): $0.57 \mathrm{~L}$, forced expiratory volume in $1 \mathrm{sec}$ (FEV1): $0.42 \mathrm{~L}]$. At the last follow-up, the patient was continuing anticoagulant treatment combined with long-term oxygen therapy to ameliorate her poor heart and lung function.

\section{Discussion}

We herein describe an intriguing case of CTEPH, which was potentially linked to chronic infection. CTEPH is defined by the following two criteria assessed after at least three months of effective anticoagulation therapy: 1) a mean pulmonary arterial pressure $>25 \mathrm{mmHg}$ with a pulmonary capillary wedge pressure of $15 \mathrm{mmHg}$; and 2) the presence of multiple chronic/organized occlusive thrombi/emboli in the elastic arteries (main, lobar, segmental, subsegmental) (1).

Although contrast-enhanced CT scans and pulmonary angiography revealed a blood clot in the lower lobe of the pulmonary artery in the present case, ventilation-perfusion scanning showed multiple wedge-shaped perfusion defects. Of the two techniques, ventilation-perfusion scanning has a greater sensitivity for diagnosing a chronic pulmonary embolism (3). In addition, Bergin et al. reported that the combination of a mosaic pattern of variable attenuation with disparity in the size of the segmental vessels on high-resolution 
a
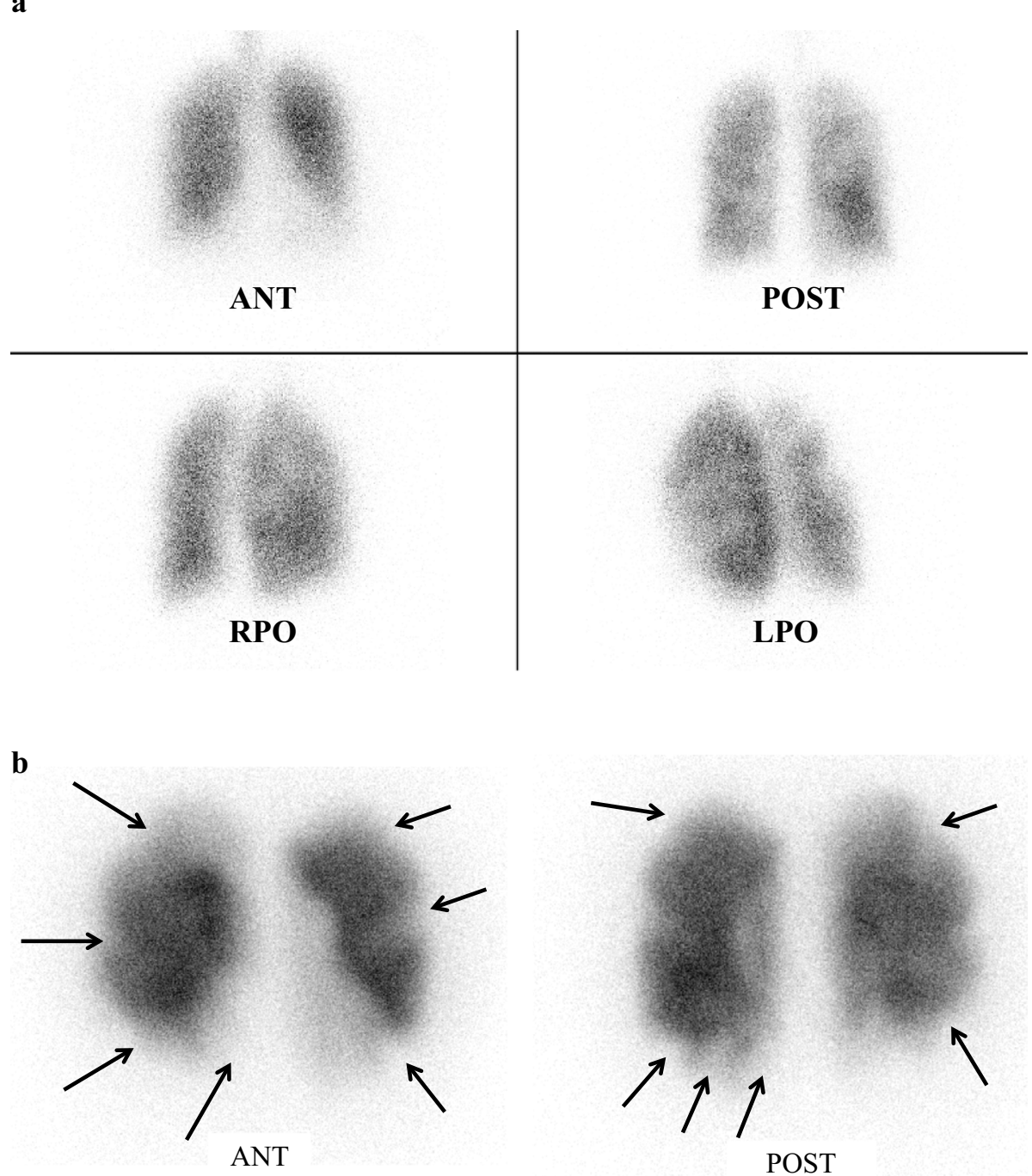

$\mathrm{R}$

$\mathrm{L}$

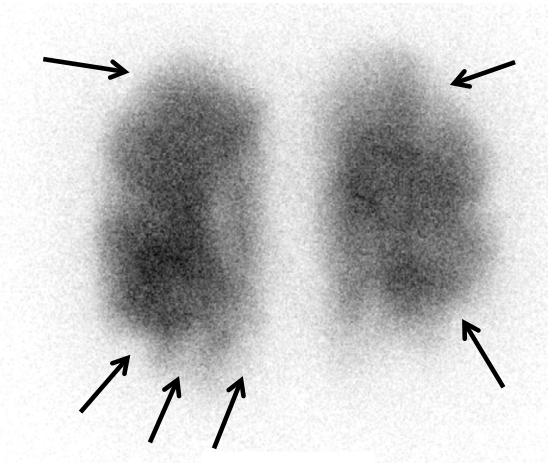

POST

L

R
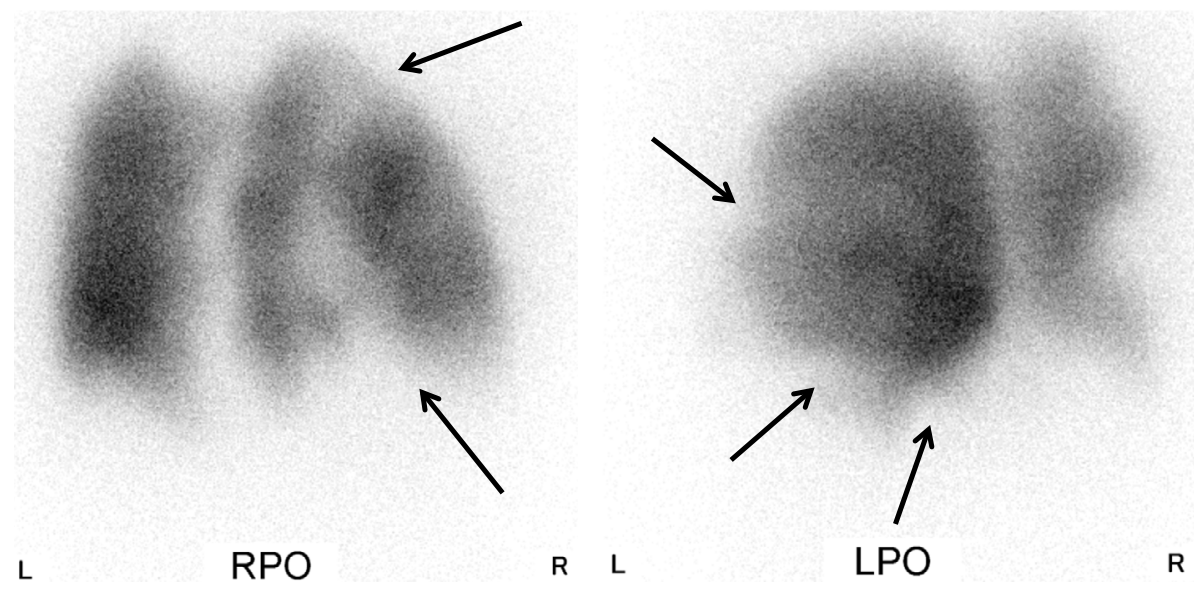

Figure 3. Ventilation-perfusion lung scintigraphy. (a) Ventilation scanning results were nearly normal. (b) Perfusion scanning demonstrated multiple perfusion defects (arrows).

CT could reliably distinguish patients with chronic pulmonary thromboembolism from those with other pulmonary diseases, including primary pulmonary arterial hypertension (4). For this reason, the findings from the CT scans and pulmonary angiography in this case report were considered to be compatible with CTEPH.

In most CTEPH cases, arterial blood gas analyses reveal hypoxemia and hypercapnia. The results of an arterial blood gas analysis from the present case showed respiratory acidosis and were indicative of chronic respiratory failure with hypoventilation. Indeed, the pulmonary function tests conducted one year prior to this hospitalization showed a low VC and FEV1.

CTEPH is generally considered to result from the forma- 

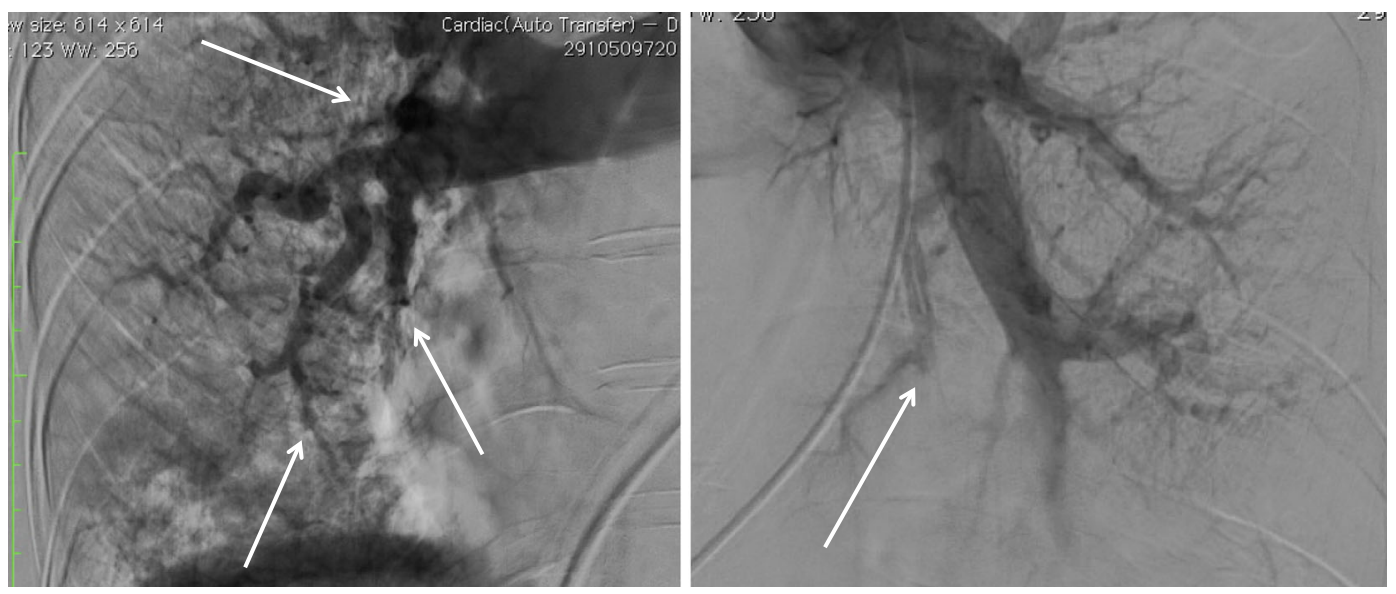

Figure 4. Pulmonary angiography showed abrupt narrowing of both segmental arteries (arrows).

tion of single or recurrent pulmonary emboli after deep vein thrombosis. However, the pathogenesis of CTEPH is not solely attributable to dysfunction in the coagulation system, and its precise etiology remains unclear because CTEPH patients do not necessarily have a history of symptomatic acute pulmonary embolism or deep vein thrombosis $(5,6)$. One of the key processes associated with CTEPH is aberrant thrombus resolution, which leads to the progression of pulmonary vascular remodeling. Therefore, mechanical obstruction of the pulmonary arteries is likely to be involved, and it has been established that abnormalities of thrombosis and thrombolysis promote the development of CTEPH. Furthermore, immunological and/or inflammatory mechanisms may induce pathological remodeling of major and minor pulmonary vessels (1). As a result, the inflammatory states could contribute to persistent obstruction by impairing the pulmonary vascular cell function, thus playing a role in the pathophysiology of CTEPH (7). Indeed, previous reports have suggested that the prevalence of inflammatory disease is higher in CTEPH patients (8-10). In this case report, no episodes of acute pulmonary embolism or deep venous thrombosis, and no classic risk factors for thrombosis were documented. In addition, the patient's HLA type was not HLAB*5201, which has been associated with CTEPH (11). A unique aspect of this case was the occurrence of five episodes of pneumonia requiring hospitalization over the previous five years. The lung architecture of the right lower lobe was disrupted due to an unspecified lung disease during childhood. Therefore, it is likely that this patient was susceptible to recurrent lung infections. In addition, the blood tests showed a slightly elevated CRP level $(0.5-3.5 \mathrm{mg} / \mathrm{dL})$ even in the absence of infection symptoms. This indicates that the patient had existing subclinical chronic inflammation. Due to the presence of chronic inflammation, differential diagnoses were investigated, including arteritis, collagen vascular diseases, malignancy, and parasitic infections. The possibility of arteritis was excluded because contrastenhanced CT and coronary angiography showed that the aorta and its major tributaries were normal.

The positivity of ANA may be indicative of collagen vas- cular disease, including mixed connective tissue disease, systemic lupus erythematosus, systemic sclerosis, and Sjögren's syndrome. The clinical features and laboratory data in the present case, together with the low ANA titer, did not fulfill the criteria for these diseases. In addition, a low titer of ANA has been detected in healthy individuals (12). Therefore, this was considered to be a nonspecific finding.

According to the CT scans, primary sarcomas of the pulmonary artery and other carcinomas, such as breast, liver and renal, which are recognized as a cause of tumor-related emboli, were not present. Parasitic infection was excluded because no clinical abnormalities, such as eosinophilia, were detected, and the patient had not left the country for over a decade. Therefore, in this case, chronic low-level inflammation was likely to be associated with subclinical infection in the disrupted lung, without obvious symptoms. Other case reports have also suggested that a relationship exists between CTEPH and infectious conditions (10). Moreover, Wynants et al. indicated that CRP was able to enhance the mitogenic activity of smooth muscle cells, inflammatory cell adhesion to endothelial cells, and secretion of endothelin-1 and von Willebrand factor (vWF) (7). Bisoendial et al. reported that an infusion of recombinant CRP to healthy individuals induced a marked elevation in the levels of vWF, prothrombin, D-dimer, and plasminogen activator inhibitor type 1 (13). According to these data, CRP may play a role in the development of CTEPH.

A noteworthy feature of the present case was the detection of Staphylococcus aureus infection in the sputum cultures from three of five previous episodes of pneumonia. Bonderman et al. proposed that staphylococcal infection delays thrombus resolution and promotes fibrotic vascular remodeling in vivo. The authors also suggested that bacterial DNA, which represents the remnants of previous infections, was a more important cause than active Staphylococcus aureus. Other papers have reported that the placement of ventriculoatrial (VA) shunts and pacemakers can lead to infection, in particular with Staphylococci species, which is a risk factor for CTEPH $(8,14,15)$. Interestingly, even in a 
rare case describing CTEPH complicated by a brain abscess, Staphylococcus aureus was the responsible bacterium (16).

In conclusion, this unique case report suggests that asymptomatic chronic inflammation, especially due to Staphylococcal species, could play a role in the development of CTEPH.

The authors state that they have no Conflict of Interest (COI).

\section{References}

1. Wilkens H, Lang I, Behr J, et al. Chronic thromboembolic pulmonary hypertension (CTEPH): updated Recommendations of the Cologne Consensus Conference 2011. Int J Cardiol 154(Suppl 1): S54-S60, 2011.

2. Lang IM, Pesavento R, Bonderman D, Yuan JX. Risk factors and basic mechanisms of chronic thromboembolic pulmonary hypertension: a current understanding. Eur Respir J 41: 462-468, 2013.

3. Tunariu N, Gibbs SJ, Win Z, et al. Ventilation-perfusion scintigraphy is more sensitive than multidetector CTPA in detecting chronic thromboembolic pulmonary disease as a treatable cause of pulmonary hypertension. J Nucl Med 48: 680-684, 2007.

4. Bergin CJ, Rios G, King MA, Belezzuoli E, Luna J, Auger WR. Accuracy of high-resolution CT in identifying chronic pulmonary thromboembolic disease. Am J Roentgenol 166: 1371-1377, 1996.

5. Lang IM. Chronic thromboembolic pulmonary hypertension: not so rare after all. N Engl J Med 350: 2236-2238, 2004.

6. Hoeper MM, Mayer E, Simonneau G, Rubin LJ. Chronic thromboembolic pulmonary hypertension. Circulation 113: 2011-2020, 2006.

7. Wynants M, Quarck R, Ronisz A, et al. Effects of C-reactive pro- tein on human pulmonary vascular cells in chronic thromboembolic pulmonary hypertension. Eur Respir J 40: 886-894, 2012.

8. Humbert M. Pulmonary arterial hypertension and chronic thromboembolic pulmonary hypertension: pathophysiology. Eur Respir Rev 19: 59-63, 2010.

9. Bonderman D, Skoro-Sajer N, Jakowitsch J, et al. Predictors of outcome in chronic thromboembolic pulmonary hypertension. Circulation 115: 2153-2158, 2007.

10. Bonderman D, Jakowitsch J, Adlbrecht $\mathrm{C}$, et al. Medical conditions increasing the risk of chronic thromboembolic pulmonary hypertension. Thromb Haemost 93: 512-516, 2005.

11. Tanabe N, Kimura A, Amano S, et al. Association of clinical features with HLA in chronic pulmonary thromboembolism. Eur Respir J 25: 131-138, 2005.

12. Mahler M, Ngo JT, Schulte-Pelkum J, Luettich T, Fritzler MJ. Limited reliability of the indirect immunofluorescence technique for the detection of anti-Rib-P antibodies. Arthritis Res Ther 10: R 131, 2008.

13. Bisoendial RJ, Kastelein JJ, Levels JH, et al. Activation of inflammation and coagulation after infusion of C-reactive protein in humans. Circ Res 96: 714-716, 2005.

14. Bonderman D, Jakowitsch J, Redwan B, et al. Role for staphylococci in misguided thrombus resolution of chronic thromboembolic pulmonary hypertension. Arterioscler Thromb Vasc Biol 28: 678-684, 2008.

15. Amelot A, Bouazza S, George B, Bresson D. Causative role of infection in chronic non-thromboembolic pulmonary hypertension following ventriculo-atrial shunt. $\mathrm{Br}$ J Neurosurg 28: 559-561, 2014.

16. Lakshmi K, Santhanam R, Chitralekha S. Brain abscess as a complication of chronic thromboembolic pulmonary hypertension: a rare case report. J Clin Diagn Res 7: 2027-2028, 2013.

(C) 2016 The Japanese Society of Internal Medicine http://www.naika.or.jp/imonline/index.html 